\title{
BIOSIGNALS EVENTS DETECTION A Morphological Signal-independent Approach
}

\author{
Rui Santos ${ }^{1}$, Joana Sousa ${ }^{2}$, Borja Sañudo ${ }^{3}$, Carlos J. Marques ${ }^{4,5}$ and Hugo Gamboa ${ }^{1,2}$ \\ ${ }^{1}$ Physics Department, FCT-UNL, Lisbon, Portugal \\ ${ }^{2}$ PLUX-Wireless Biosignals, S.A., Lisbon, Portugal \\ ${ }^{3}$ Department of Physical Education and Sport, University of Seville, Seville, Spain \\ ${ }^{4}$ Faculty of Human Kinetics at the Technical University of Lisbon, Lisbon, Portugal \\ ${ }^{5}$ Physical Therapy and Rehabilitation Department at the Schön Klinik Hamburg Eilbek, Hamburg, Germany
}

Keywords: Biosignals, Signal-processing, Events, Detection and Identification, Signal-independent.

\begin{abstract}
This study presents a signal-independent algorithm, which detects significant events in a biosignal, without previous knowledge or specific pre-processing steps. From a morphological analysis, the algorithm computes the instants when the most significant standard deviation discontinuities occur. An iterative optimization step is then applied. This assures that a minimal error is achieved when modeling the signal segments (between the detected instants) with a polynomial regression. The detection scale can be modified by an optional input scale factor. An objective algorithm performance evaluation procedure was designed, and applied on two types of synthetic signals, for which the events instants were previously known. An overall mean error of $20.32( \pm 16.01)$ samples between the detected and the real events show the high accuracy of the proposed algorithm. The algorithm was also applied on accelerometry and electromyography raw signals collected in different experimental scenarios. The fact that this approach does not require any previous knowledge and the good level of accuracy represents a relevant contribution in events detection and biosignal analysis.
\end{abstract}

\section{INTRODUCTION}

Automated techniques for generating, acquiring and storing data from scientific measurements have become increasingly precise and powerful. However, there is still a practical need to improve tools for signal pattern recognition and interpretation systems, in which the detection of specific events and the automatic signal segmentation must be one of the first processing procedures (Ciaccio et al., 1993). An event is broadly defined as the change in state of the system under study (Ciaccio et al., 1993). Biosignals are often characterized by oscillations at specific frequencies and contaminated by in-band noise, which is both periodic and random (Clifford, 2006). Identifying the underlying biosignal and its specific events among trivial changes can become extremely difficult.

The accurate onset determination of electromyographic (EMG) activity is an application of events detection algorithms. A comparative study regarding several methods for EMG signals onset detection, including those proposed by Hodges (Hodges and Bui, 1996) and Bonato (Bonato et al., 1998), is reported by
Staude (Staude et al., 2001). Another possible application is related to the detection of general transient events. Abrupt changes or discontinuities encountered in biosignals may be symptomatic of functional disorders (Sankur et al., 1996). The ability to detect them has great importance to the medical prediction process, as well as in sport and rehabilitation research fields.

In the present work we've developed an algorithm for biosignals events detection. Based on a morphological analysis, it is able to identify time domain specific shape parameters that can clearly distinguish those events from the complete observed signals. The algorithm's design follows a signal-independent approach, requiring also no prior information or preprocessing steps, but allowing the user to control the detail on the event detection by optionally changing a specific scale factor.

The algorithm was applied on acceleration and EMG digital raw signals. An objective performance evaluation procedure was designed and applied on synthetic signals, for which the events were known. 


\section{MATERIALS AND ACQUISITION METHODS}

\subsection{Synthetic Signals}

Synthetic signals were constructed by concatenating sections with predefined mean and standard deviation (STD) values (referenced as zones). The mean values where given by float numbers randomly sampled from a standard normal distribution and then multiplied by a factor $m$. The STD values were obtained by multiplying a random number sampled from a uniform distribution, within the interval $[0,1]$ by a factor $s$. Between those zones transition events with known randomly selected starting and ending points were also considered. Minimal values for mean and STD differences between successive zones were imposed and assigned to the $m_{-} d$ and $s d \_d$ variables, respectively. Two different types of synthetic signals were defined according to the parameters in Table 1.

Table 1: Synthetic signals parameters.

\begin{tabular}{ccc}
\hline Synthetic signal & Type I & Type II \\
\hline \hline$m$ & 8 & 0.05 \\
\hline$s$ & 0.1 & 1 \\
\hline$m_{\_} d$ & 5 & 0.1 \\
\hline$s d \_d$ & 0.05 & 0.5 \\
\hline Number of events & 10 & 5 \\
\hline
\end{tabular}

\subsection{Data Acquisition}

The EMG and acceleration signals were acquired, respectively, using surface bipolar EMG sensors (emgPLUX) and triaxial accelerometers (xyzPLUX), connected to a bioPLUX research unit (PLUX, 2007). Signals were sampled at frequency of $1000 \mathrm{~Hz}$.

For acquiring acceleration signals drop jumps from a $40 \mathrm{~cm}$ elevated platform to the ground level were executed. The accelerometer was placed next to the knee (at the tibial condyle level) and orientated so its $\mathrm{x}$ axis was pointing upward. Only the signal acquired from the $\mathrm{z}$ axis (the acceleration in the Medial-Lateral axis) was considered. The testing protocol that was followed to obtain EMG signals aimed at accessing the performance of an emergency brake in a car simulator (Marques et al., 2011). From the set of signals acquired in the context of that study we consider the EMG records from the Tibialis anterior muscle during the foot transition from the accelerator to the brake pedal.

\section{EVENTS DETECTION ALGORITHM}

\subsection{Mathematical Formalism}

For a given signal defined as a time series, $x(t)$, with $t=1,2, \ldots, L$, a set of regions is created by slicing the signal. Considering $E$ as the total number of events, a general event slicing signal regions is denoted by $e_{i}, i=1,2, \ldots, E$. The complete modeled signal is expressed as defined in equation 1 , for which further notation is described below:

$$
\hat{x}(t)=\sum_{i=0}^{E-1} Q\left(\frac{t}{e_{i+1}-e_{i}}-e_{i}\right) M\left(t-e_{i}, A_{i}, \varepsilon\right)
$$

$Q(t)$ is an unit pulse function expressed by:

$$
Q(t)= \begin{cases}1 & \text { if } 0 \leq t \leq 1 \\ 0 & \text { otherwise }\end{cases}
$$

$M(t, A, \varepsilon)$ is a polynomial regression model defined by:

$$
M(t, A, \varepsilon)=A(t)+\varepsilon
$$

where $A$ is the matrix with $n$ polynomial parameters for a given signal region $\left(A(t)=a_{0}+a_{1} t+\ldots .+a_{n} t^{n}\right)$ and $\varepsilon$ is an error term that is assumed to follow a normal distribution $\left(\varepsilon=N\left(\mu, \sigma^{2}\right)\right)$ with $\mu=0$. Increasing the order of $M$ makes the polynomial regression to better model the signal, decreasing the Mean Squared Error (MSE), but also renders this algorithm less sensitive to abrupt changes within that signal.

After modeling the signal as described above, each one of the $E-1$ signal segments can be described as:

$$
\hat{x}^{i}(t)=\left.\hat{x}(t)\right|_{t \in\left[e_{i}, e_{i+1}\right]}
$$

\subsection{Implementation}

\subsubsection{Events Detection}

The get_events algorithm is the base function to identify the signal events (Figure 1). Beyond the signal, it can receive other parameters, by input, in order to run auxiliary functions responsible for each of the processing steps described below.

From a raw signal, the get_slices_std function divides the signal into slices with a defined length (slices_length). This function returns an array containing the sequence of the successive slice's STD. The STD sequence first derivative is then calculated and low-pass filtered by applying a smoothing filter, in which the number of points considered into the moving average window depends on the length of the sequence multiplied by the smooth_factor 


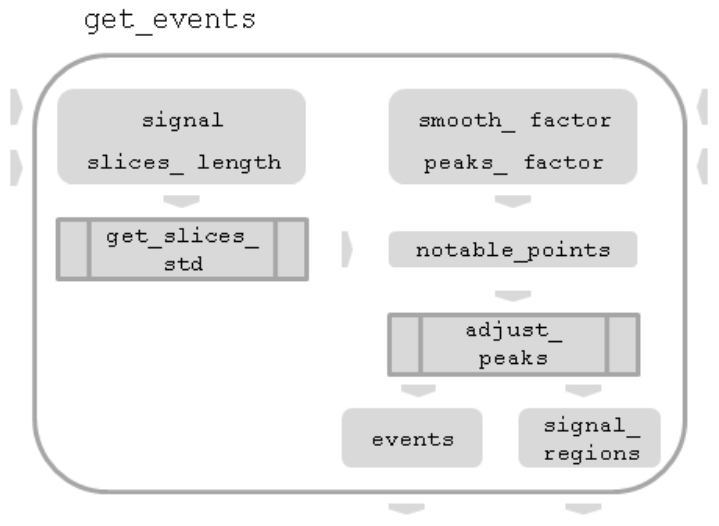

Figure 1: get_events algorithm flowchart diagram.

value (Figure 1). From the filtered sequence, the signal notable points are computed from the local maximums/minimums found above/below a specific threshold. The former is defined multiplying the absolute sequence maximum/minimum by the peaks_factor input parameter (Figure 1).

The adjust_peaks function is then applied. For each peak this function considers the signal slice beginning in each notable point and then applies the get_slices_std function on that signal segment (with a fixed slices_length of 5 samples). If a maximum peak is considered, it is then replaced by the point that maximizes the difference on the computed STD sequence. For minimums the procedure is analogous. This processing step ensures a more accurate events detection, with a minimum error of 5 samples. At the end, the get_events algorithm returns the detected events and an array with the successive signal regions between those events.

\subsubsection{Optimization}

The optimization processing step is based on an iterative change into the input parameters of the get_events algorithm (slices_length, smooth_factor and peaks_factor). The respective ranges depend on a scale factor, defined with base on the signal's length. It can alternatively be an input of the optimization algorithm, allowing to increase or decrease the level of detail (the number of detected events). For each set of parameters the get_events algorithm is applied. Either the solutions that have the minimum total MSE, considering all the signal segments $\left(\hat{x}^{i}(t)\right.$ in equation 4$)$ modeled as 1 st order polynomials, or the maximum total standard deviation differences between those segments are selected in the same step. From those, the one for which the number of events is maximal is selected as the optimal solution.

\section{RESULTS AND DISCUSSION}

\subsection{Algorithm Performance Evaluation}

The developed algorithm was applied on a total of 25 Type I and 25 Type II synthetic signals, for which examples of graphic representations, including marks on the detected events, are given in Figure 2. The default scale factor was used. For each synthetic signal the detection mean error was computed by evaluating the mean of the differences between the detected events and the closer real event. An overall mean error of $20.32( \pm 16.01)$ samples show the high accuracy of the proposed algorithm.
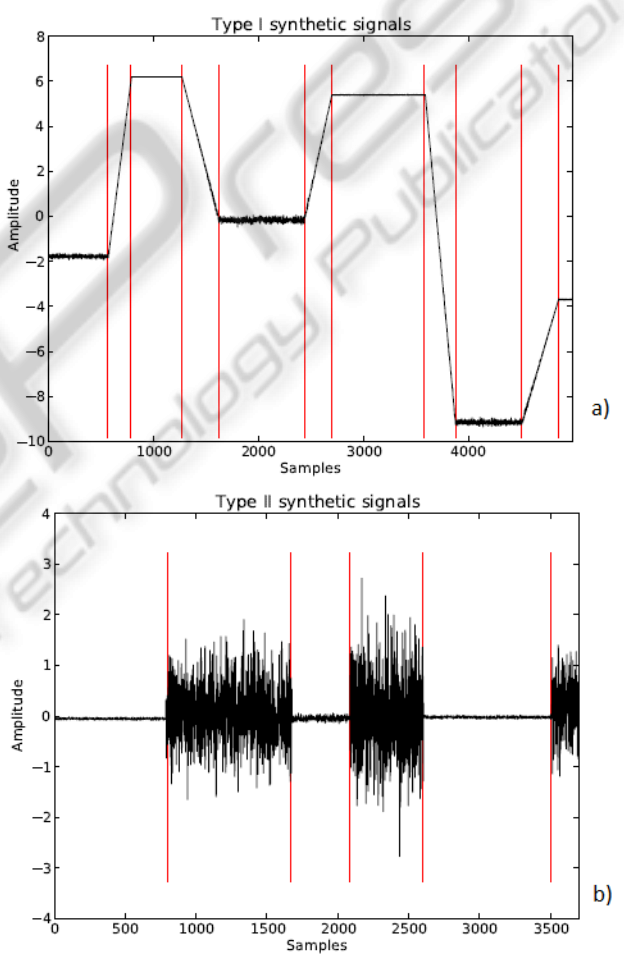

Figure 2: Synthetic signals results: a) Type I and b) Type II. The detected events are represented as vertical red marks.

\subsection{Application to Biosignals}

The default mode algorithm application to knee acceleration signals (Figure 3a) ) marks the drop jump event by selecting mostly the landing phase record, which is characterized for the greater instability and amplitude within the signal. A more accurate description of the acceleration pattern is achieved with a proper scale factor. The results presented in Figure $3 \mathrm{~b}$ ), with a scale factor of 0.02 , allow to distinguish the knee acceleration while preparing the jump from the platform, the flying phase (with a slight and longer 

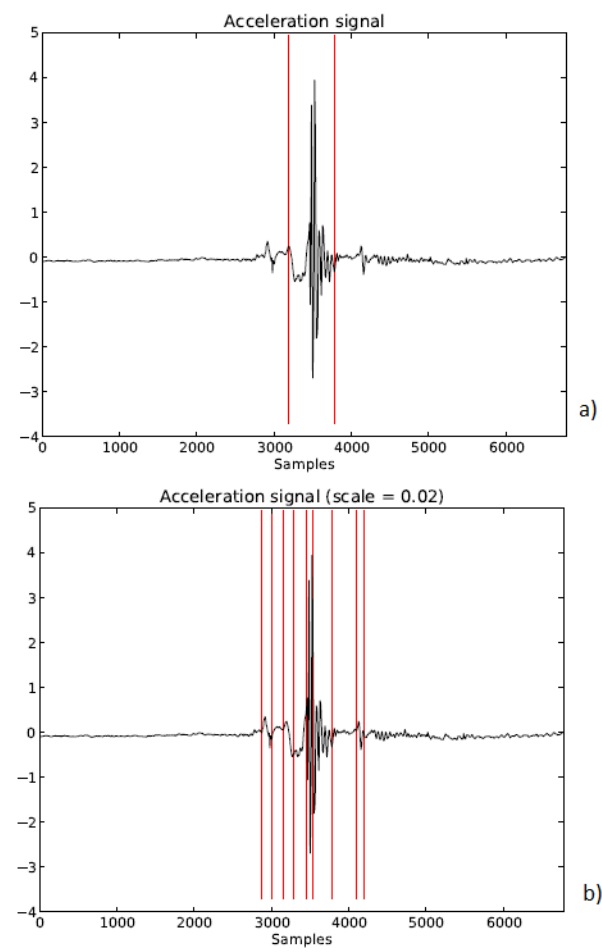

Figure 3: Acceleration signal results considering: a) default scale factor and b) a scale factor of 0.02 .

acceleration instability) and the landing phase, where the instability increases significantly for a short time period. The time spent recovering the knee stability can also be estimated by accessing the posterior signal segments. When applied to EMG signals, the default mode events detection allowed a clear distinction between activation and rest phases with an acute onset and offset detection. Being able to detect multiple events and requiring no EMG specific pre-processing steps, this algorithm presents advantages when compared with the standard onset detection techniques (Staude et al., 2001).

\section{CONCLUSIONS AND FUTURE WORK}

The proposed algorithm performs an efficient events detection within a signal. Its versatile design allows the application in different signals, without previous knowledge on their statistical characteristics and the adjustment of a scale factor to achieve different detail levels in specific applications. The added accuracy and objectivity of this algorithm when compared with the standard visual inspection also represents an advance in events detection from biosignals analysis.

In future work there is the intention of apply- ing the algorithm to a wider range of biosignals and evaluate its performance when compared with signal specific processing techniques. Its integration into a real-time processing tool is already under development. Preliminary results point out the application's ability to detect the events in real-time, without a significant loss of accuracy.

\section{ACKNOWLEDGEMENTS}

This work was partially supported by National Strategic Reference Framework (NSRF-QREN) under projects "LUL" and "Affective Mouse", and Seventh Framework Programme (FP7) program under project ICT4Depression, whose support the authors gratefully acknowledge.

\section{REFERENCES}

Bonato, P., D'Alessio, T., and Knaflitz, M. (1998). A statistical method for the measurement of muscle activation intervals from surface myoelectric signal during gait. Biomedical Engineering, IEEE Transactions on, 45(3):287-299.

Ciaccio, E., Dunn, S., and Akay, M. (1993). Biosignal pattern recognition and interpretation systems. Engineering in Medicine and Biology Magazine, IEEE, 12(3):89-95.

Clifford, G. (2006). A novel framework for signal representation and source separation: Applications to filtering and segmentation of biosignals. Journal of Biological Systems, 14(2):169-184.

Hodges, P. and Bui, B. (1996). A comparison of computer-based methods for the determination of onset of muscle contraction using electromyography. Electroencephalography and Clinical Neurophysiology/Electromyography and Motor Control, 101(6):511-519.

Marques, C. J., Gamboa, H., Lampe, F., Barreiros, J., and Cabri, J. (2011). Muscle activation thresholds before and after total knee arthoplasty - protocol of a randomized comparison of minimally invasive vs. standard approach. In Proceedings of the International Conference on Bio-inspired Systems and Signal Processing.

PLUX (2007). PLUX - Wireless Biosignals, S.A. [online] Available at: http://plux.info/ [Accessed 5 September 2011].

Sankur, B., Güler, E. C., and Kahya, Y. (1996). Multiresolution biological transient extraction applied to respiratory crackles. Computers in biology and medicine, 26(1):25-39.

Staude, G., Flachenecker, C., Daumer, M., and Wolf, W. (2001). Onset detection in surface electromyographic signals: a systematic comparison of methods. EURASIP Journal on Applied Signal Processing, 2001(1):67-81. 